Bull. Austral. Math. Soc.

$47 \mathrm{H} 10,47 \mathrm{H} 09$

VOL. 69 (2004) [1-18]

\title{
COMMON FIXED POINTS OF TWO NONEXPANSIVE MAPPINGS IN BANACH SPACES
}

\author{
TOMONARI SUZUKI
}

\begin{abstract}
In this paper, we discuss a necessary and sufficient condition for common fixed points of two nonexpansive mappings. We then prove a convergence theorem to a common fixed point. Finally, we discuss the existence of a nonexpansive retraction onto the set of common fixed points of nonexpansive mappings. In these theorems, we do not assume the strict (uniform) convexity of the norm of the Banach space.
\end{abstract}

\section{INTRODUCTION}

A mapping $T$ on a closed convex subset $C$ of a Banach space $E$ is called a nonexpansive mapping if $\|T x-T y\| \leqslant\|x-y\|$ for all $x, y \in C$. We denote by $F(T)$ the set of fixed points of $T$. We know that $F(T)$ is nonempty if $E$ is uniformly convex and $C$ is bounded; see Browder [4] and Göhde [15]. Kirk [21] extended these result to the case that $C$ is weakly compact and has normal structure. A subset $F$ of $C$ is called a nonexpansive retract of $C[5,13]$ if either $F$ is empty, or there exists a retraction from $C$ onto $F$ which is a nonexpansive mapping. In 1970, Bruck [5] shows that if $E$ is reflexive and strictly convex, then $F(T)$ is a nonexpansive retract of $C$; see also [6]. The existence of common fixed point for families of nonexpansive mappings was established by several authors; see Belluce and Kirk [2, 3], Browder [4], DeMarr [9], Lim [24] and others. In 1974, Bruck [7] prove the following generalisation of these common fixed point theorems.

THEOREM 1. (Bruck [7].) Suppose a closed convex subset $C$ of a Banach space has both the fixed point property and the conditional fixed point property for nonexpansive mappings, and $C$ is either weakly compact, or bounded and separable. Then for any commuting family $S$ of nonexpansive mappings on $C$, the set of common fixed points of $S$ is a nonempty nonexpansive retract of $C$.

Convergence theorems for nonexpansive mappings using Mann's iteration process ([27]) also have been studied by several authors; see Edelstein [11], Edelstein and O'Brien [12], Groetsch [17], Ishikawa [18], Krasnoselskii [22], Opial [28], Reich [30] and others. For example, Edelstein and O'Brien [12] proved the following.

Received 10th February, 2003

The author is supported in part by Grants-in-Aid for Scientific Research, Japan Society for the Promotion of Science.

Copyright Clearance Centre, Inc. Serial-fee code: 0004-9727/04 \$A2.00+0.00. 
Theorem 2. (Edelstein and O'Brien [12].) Let $E$ be a Banach space with the Opial property and let $T$ be a nonexpansive mapping on a weakly compact convex subset $C$ of $E$. Let $x_{1} \in C$ and define a sequence $\left\{x_{n}\right\}$ in $C$ by $x_{n+1}=\alpha T x_{n}+(1-\alpha) x_{n}$ for $n \in \mathbb{N}$, where $\alpha$ is a constant number in $(0,1)$. Then $\left\{x_{n}\right\}$ converges weakly to a fixed point of $T$.

Ishikawa [18] proved the following strong convergence theorem.

THEOREM 3. (Ishikawa [18].) Let $T$ be a nonexpansive mapping on a compact convex subset $C$ of a Banach space $E$. Let $x_{1} \in C$ and define a sequence $\left\{x_{n}\right\}$ in $C$ by $x_{n+1}=\alpha_{n} T x_{n}+\left(1-\alpha_{n}\right) x_{n}$ for $n \in \mathbb{N}$, where $\left\{\alpha_{n}\right\}$ is a sequence in $[0,1]$ satisfying $\lim \sup _{n} \alpha_{n}<1$ and $\sum_{n=1}^{\infty} \alpha_{n}=\infty$. Then $\left\{x_{n}\right\}$ converges strongly to a fixed point of $T$.

Convergence theorems for families of nonexpansive mappings are proved in Atsushiba and Takahashi [1], Crombez [8], Ishikawa [19], Linhart [26] and others.

In this paper, we discuss a necessary and sufficient condition for common fixed points of two nonexpansive mappings. We then prove a convergence theorem to a common fixed point. Finally, we discuss the existence of a nonexpansive retraction onto the set of common fixed points of the nonexpansive mappings. In these theorems, we do not assume the strict (uniform) convexity of the norm of the Banach space.

\section{Preliminaries}

Throughout this paper, we denote by $\mathbb{N}$ and $\mathbb{R}$ the set of positive integers and real numbers, respectively. We put $N(k, \ell)=\{i \in \mathbb{N}: k \leqslant i \leqslant \ell\}$ and $N(k, \infty)=\{i \in \mathbb{N}$ : $k \leqslant i\}$ for $k, \ell \in \mathbb{N}$. For an arbitrary set $A$, we also denote by $\sharp A$ the number of elements of $A$. Let $E$ be a Banach space. In this paper, we assume that a Banach space $E$ is real. We denote by $E^{*}$ the dual space of $E$. $E$ is said to have the Opial property ([28]) if for each weakly convergent sequence $\left\{x_{n}\right\}$ in $E$ with weak limit $z_{0}$,

$$
\liminf _{n \rightarrow \infty}\left\|x_{n}-z_{0}\right\|<\liminf _{n \rightarrow \infty}\left\|x_{n}-x\right\|
$$

holds for all $x \in E$ with $x \neq z_{0}$. All Hilbert spaces, all finite dimensional Banach spaces and $\ell^{p}(1 \leqslant p<\infty)$ have the Opial property. A Banach space with a duality mapping which is weakly sequentially continuous also has the Opial property; see [16]. See also [23], [25], [29] and [31]. We know that every separable Banach space can be equivalently renormed so that it has the Opial property; see [10]. We also know the following.

Proposition 1. Let $E_{1}, E_{2}, \ldots, E_{k}$ be Banach spaces with norm $\|\cdot\|_{1}$, $\|\cdot\|_{2}, \ldots,\|\cdot\|_{k}$, respectively. Let $p$ be a constant in $[1, \infty)$ and put $E=E_{1} \times E_{2} \times \cdots \times E_{k}$, where the norm of $E$ is given by

$$
\left\|\left(x_{1}, x_{2}, \ldots, x_{k}\right)\right\|=\left(\left\|x_{1}\right\|_{1}^{p}+\left\|x_{2}\right\|_{2}^{p}+\cdots+\left\|x_{k}\right\|_{k}^{p}\right)^{1 / p}
$$

for all $\left(x_{1}, x_{2}, \ldots, x_{k}\right) \in E$. Then the following are equivalent: 
(i) E has the Opial property;

(ii) each $E_{j}$ has the Opial property.

Proof: If a sequence $\left\{x_{n}\right\}$ in $E_{j}$ converges weakly to $z$, then a sequence

$$
\left\{\left(0,0, \ldots, 0, \stackrel{(j)}{x_{n}}, 0, \ldots, 0\right)\right\}
$$

in $E$ converges weakly to

$$
\{(0,0, \ldots, 0, \stackrel{(j)}{z}, 0, \ldots, 0)\}
$$

Using this fact, we can prove easily (i) implies (ii). Conversely, let $\left\{x^{(n)}\right\}$ be a sequence in $E$ which converges weakly to $z$, and let $w$ belong to $E \backslash\{z\}$. We put $x^{(n)}=\left(x_{1}^{(n)}, x_{2}^{(n)}, \ldots, x_{k}^{(n)}\right)$ for $n \in \mathbb{N}, z=\left(z_{1}, z_{2}, \ldots, z_{k}\right)$ and $w=\left(w_{1}, w_{2}, \ldots, w_{k}\right)$. Since $\left\{x^{(n)}\right\}$ is a bounded sequence, there exists a subsequence $\left\{x^{\left(n_{i}\right)}\right\}$ of $\left\{x^{(n)}\right\}$ such that

$$
\liminf _{n \rightarrow \infty}\left\|x^{(n)}-w\right\|^{p}=\lim _{i \rightarrow \infty}\left\|x^{\left(n_{i}\right)}-w\right\|^{p},
$$

and that the limit of $\left\{\left\|x_{j}^{\left(n_{i}\right)}-z_{j}\right\|_{j}\right\}$ exist for all $j \in N(1, k)$. Since $E_{j}$ for $j \in N(1, k)$ have the Opial property,

$$
\lim _{i \rightarrow \infty}\left\|x_{j}^{\left(n_{i}\right)}-z_{j}\right\|_{j}^{p} \leqslant \liminf _{i \rightarrow \infty}\left\|x_{j}^{\left(n_{i}\right)}-w_{j}\right\|_{j}^{p}
$$

hold for all $j \in N(1, k)$, and

$$
\lim _{i \rightarrow \infty}\left\|x_{\ell}^{\left(n_{i}\right)}-z_{\ell}\right\|_{\ell}^{p}<\liminf _{i \rightarrow \infty}\left\|x_{\ell}^{\left(n_{i}\right)}-w_{\ell}\right\|_{\ell}^{p}
$$

holds for some $\ell \in N(1, k)$ because $z \neq w$. Therefore we have

$$
\begin{aligned}
\liminf _{n \rightarrow \infty}\left\|x^{(n)}-z\right\|^{p} & \leqslant \lim _{i \rightarrow \infty}\left\|x^{\left(n_{i}\right)}-z\right\|^{p} \\
& =\sum_{j=1}^{k} \lim _{i \rightarrow \infty}\left\|x_{j}^{\left(n_{i}\right)}-z_{j}\right\|_{j}^{p} \\
& <\sum_{j=1}^{k} \liminf _{i \rightarrow \infty}\left\|x_{j}^{\left(n_{i}\right)}-w_{j}\right\|_{j}^{p} \\
& \leqslant \lim _{i \rightarrow \infty}\left\|x^{\left(n_{i}\right)}-w\right\|^{p} \\
& =\liminf _{n \rightarrow \infty}\left\|x^{(n)}-w\right\|^{p}
\end{aligned}
$$

and hence

$$
\liminf _{n \rightarrow \infty}\left\|x^{(n)}-z\right\|<\liminf _{n \rightarrow \infty}\left\|x^{(n)}-w\right\| .
$$

This completes the proof.

The following lemma plays an important role in the proof of Theorem 4. 
Lemma 1. Let $A_{1}, A_{2}, A_{3}, \ldots, A_{k}$ be subsets of $\mathbb{N}^{2}$. Put $A_{j}(n)=N(1, n)^{2} \cap A_{j}$ for $n \in \mathbb{N}$ and $j \in N(1, k)$, and put

$$
\alpha=\sum_{j=1}^{k} \liminf _{n \rightarrow \infty} \frac{\sharp A_{j}(n)}{n^{2}}-k+1 .
$$

Suppose $\alpha>0$. Then

holds and

$$
\liminf _{n \rightarrow \infty} \frac{\sharp\left(\bigcap_{j=1}^{k} A_{j}(n)\right)}{n^{2}} \geqslant \alpha
$$

hold for all $n \in \mathbb{N}$.

$$
N(n, \infty)^{2} \cap\left(\bigcap_{j=1}^{k} A_{j}\right) \neq \emptyset
$$

Proof: From the assumption, we have

$$
\begin{aligned}
\liminf _{n \rightarrow \infty} \frac{\sharp\left(\bigcap_{j=1}^{k} A_{j}(n)\right)}{n^{2}} & \geqslant \liminf _{n \rightarrow \infty} \frac{\sum_{j=1}^{k} \sharp A_{j}(n)-(k-1) \cdot n^{2}}{n^{2}} \\
& \geqslant \sum_{j=1}^{k} \liminf _{n \rightarrow \infty} \frac{\sharp A_{j}(n)}{n^{2}}-(k-1) \\
& =\alpha .
\end{aligned}
$$

Fix $n \in \mathbb{N}$. Then we can choose $m \in \mathbb{N}$ satisfying

From

$$
m \geqslant \frac{3 n}{\alpha} \text { and } \quad \frac{\sharp\left(\bigcap_{j=1}^{k} A_{j}(m)\right)}{m^{2}}>\frac{2}{3} \alpha .
$$

$$
\sharp\left(\bigcap_{j=1}^{k} A_{j}(m)\right)>\frac{2}{3} \alpha m^{2} \geqslant 2 m n>m^{2}-(m-n)^{2}=\sharp\left(N(1, m)^{2} \backslash N(n+1, m)^{2}\right),
$$

we have

$$
\begin{aligned}
& \emptyset \varsubsetneqq\left(\bigcap_{j=1}^{k} A_{j}(m)\right) \backslash\left(N(1, m)^{2} \backslash N(n+1, m)^{2}\right) \\
& =N(n+1, m)^{2} \cap\left(\bigcap_{j=1}^{k} A_{j}(m)\right) \\
& \subset N(n, \infty)^{2} \cap\left(\bigcap_{j=1}^{k} A_{j}\right) .
\end{aligned}
$$

This completes the proof. 


\section{COMMON FIXED POINT}

In this section, we discuss a necessary and sufficient condition for common fixed points of two nonexpansive mappings. Let $S$ and $T$ be nonexpansive mappings on a closed convex subset $C$ of a Banach space $E$. Then we put

$$
M(n, x)=\frac{1}{n^{2}} \sum_{i=1}^{n} \sum_{j=1}^{n} S^{i} T^{j} x
$$

for $n \in \mathbb{N}$ and $x \in C$. Note that a mapping $M(n, \cdot)$ on $C$ is nonexpansive for each $n \in \mathbb{N}$ because

$$
\|M(n, x)-M(n, y)\| \leqslant \frac{1}{n^{2}} \sum_{i=1}^{n} \sum_{j=1}^{n}\left\|S^{i} T^{j} x-S^{i} T^{j} y\right\| \leqslant \frac{1}{n^{2}} \sum_{i=1}^{n} \sum_{j=1}^{n}\|x-y\|=\|x-y\|
$$

for all $x, y \in C$.

The following theorem is one of the main results in this paper.

Theorem 4. Let $E$ be a Banach space with the Opial property and let $C$ be a weakly compact convex subset of $E$. Let $S$ and $T$ be nonexpansive mappings on $C$ with $S T=T S$. Then for $z \in C$, the following are equivalent:

(i) $z$ is a common fixed point of $S$ and $T$;

(ii) $\{M(n, z)\}$ converges weakly to $z$;

(iii) there exists a subnet $\left\{M\left(\nu_{\beta}, z\right): \beta \in D\right\}$ of a sequence $\{M(n, z)\}$ in $C$ converging weakly to $z$.

It is clear that (i) implies (ii) and (iii). We prove only (ii) implies (i) because we can similarly prove that (iii) implies (i). Before proving this, we need preliminaries. In the following lemmas and the proof of Theorem 4, we put

$$
\begin{aligned}
d & =\lim _{k \rightarrow \infty} \sup \left\{\left\|S^{i} T^{j} z-z\right\|: i \geqslant k, j \geqslant k\right\}, \\
A(n, f, \varepsilon) & =\left\{(i, j) \in N(1, n)^{2}: f\left(S^{i} T^{j} z-z\right) \leqslant \varepsilon\right\}
\end{aligned}
$$

for $n \in \mathbb{N} \cup\{\infty\}, f \in E^{*}$ and $\varepsilon>0$, and

$$
B(n, \varepsilon)=\left\{(i, j) \in N(1, n)^{2}:\left\|S^{i} T^{j} z-z\right\| \geqslant d-\varepsilon\right\}
$$

for $n \in \mathbb{N} \cup\{\infty\}$ and $\varepsilon>0$.

Lemma 2. For every $(i, j) \in(\mathbb{N} \cup\{0\})^{2}$,

$$
\left\|S^{i} T^{j} z-z\right\| \leqslant d
$$

holds. 
Proof: Fix $\left(i_{1}, j_{1}\right) \in(\mathbb{N} \cup\{0\})^{2}$. By the Hahn-Banach theorem, there exists $f \in E^{*}$ such that

$$
\|f\|=1 \quad \text { and } \quad f\left(S^{i_{1}} T^{j_{1}} z-z\right)=\left\|S^{i_{1}} T^{j_{1}} z-z\right\| .
$$

Fix $\delta>0$. Then there exists $m_{1} \in \mathbb{N}$ such that $\left\|S^{i} T^{j} z-z\right\| \leqslant d+\delta$ for $(i, j) \in N\left(m_{1}, \infty\right)^{2}$. For $n \in \mathbb{N}$ with $n>i_{1}+j_{1}+m_{1}$, we have

$$
\begin{aligned}
\left\|S^{i_{1}} T^{j_{1}} z-z\right\|= & f\left(S^{i_{1}} T^{j_{1}} z-z\right) \\
= & f\left(S^{i_{1}} T^{j_{1}} z-M(n, z)\right)+f(M(n, z)-z) \\
\leqslant & \|f\| \cdot\left\|S^{i_{1}} T^{j_{1}} z-M(n, z)\right\|+f(M(n, z)-z) \\
= & \left\|S^{i_{1}} T^{j_{1}} z-M(n, z)\right\|+f(M(n, z)-z) \\
\leqslant & \frac{1}{n^{2}} \sum_{i=1}^{n} \sum_{j=1}^{n}\left\|S^{i_{1}} T^{j_{1}} z-S^{i} T^{j} z\right\|+f(M(n, z)-z) \\
\leqslant & \frac{1}{n^{2}} \sum_{i=i_{1}+m_{1}+1}^{n} \sum_{j=j_{1}+m_{1}+1}^{n}\left\|S^{i_{1}} T^{j_{1}} z-S^{i} T^{j} z\right\| \\
& \quad+\frac{n^{2}-\left(n-i_{1}-m_{1}\right)\left(n-j_{1}-m_{1}\right)}{n^{2}} 2 \sup \{\|x\|: x \in C\} \\
& \quad+f(M(n, z)-z) \\
\leqslant & \frac{1}{n^{2}} \sum_{i=i_{1}+m_{1}+1}^{n} \sum_{j=j_{1}+m_{1}+1}^{n}\left\|z-S^{i-i_{1}} T^{j-j_{1}} z\right\| \\
& \quad+\frac{n^{2}-\left(n-i_{1}-m_{1}\right)\left(n-j_{1}-m_{1}\right)}{n^{2}} 2 \sup \{\|x\|: x \in C\} \\
& \quad+f(M(n, z)-z) \\
\leqslant & \frac{\left(n-i_{1}-m_{1}\right)\left(n-j_{1}-m_{1}\right)}{n^{2}}(d+\delta) \\
& \quad+\frac{n^{2}-\left(n-i_{1}-m_{1}\right)\left(n-j_{1}-m_{1}\right)}{n^{2}} 2 \sup \{\|x\|: x \in C\} \\
& +f(M(n, z)-z)
\end{aligned}
$$

and hence

$$
\begin{aligned}
\left\|S^{i_{1}} T^{j_{1}} z-z\right\| \leqslant & \liminf _{n \rightarrow \infty}\left(\frac{\left(n-i_{1}-m_{1}\right)\left(n-j_{1}-m_{1}\right)}{n^{2}}(d+\delta)\right. \\
& +\frac{n^{2}-\left(n-i_{1}-m_{1}\right)\left(n-j_{1}-m_{1}\right)}{n^{2}} 2 \sup \{\|x\|: x \in C\} \\
& +f(M(n, z)-z)) \\
\leqslant & +\delta .
\end{aligned}
$$

Since $\delta$ is arbitrary, we have $\left\|S^{i_{1}} T^{j_{1}} z-z\right\| \leqslant d$. 
Lemma 3. Fix $p, q \in \mathbb{N}$ and let $f \in E^{*}$ with

$$
\|f\|=1 \quad \text { and } \quad f\left(S^{p} T^{q} z-z\right)=\left\|S^{p} T^{q} z-z\right\| .
$$

Let $\delta$ be a positive real number satisfying

$$
\left\|S^{p} T^{q} z-z\right\| \geqslant d-\delta .
$$

Then

$$
\liminf _{n \rightarrow \infty} \frac{\sharp A(n, f, \varepsilon)}{n^{2}} \geqslant \frac{\varepsilon}{\varepsilon+\delta}
$$

hold for all $\varepsilon>0$.

Proof: For $(i, j) \in \mathbb{N}^{2}$, by Lemma 2, we have

$$
\left\|S^{p} T^{q} z-S^{i} T^{j} z\right\| \leqslant\left\|S^{p} T^{q} z-z\right\|+\left\|S^{i} T^{j} z-z\right\| \leqslant 2 d
$$

and hence

$$
\begin{aligned}
f\left(S^{i} T^{j} z-z\right) & =f\left(S^{i} T^{j} z-S^{p} T^{q} z\right)+f\left(S^{p} T^{q} z-z\right) \\
& \geqslant-\|f\| \cdot\left\|S^{i} T^{j} z-S^{p} T^{q} z\right\|+d-\delta \\
& \geqslant-d-\delta .
\end{aligned}
$$

For $(i, j) \in N(p+1, \infty) \times N(q+1, \infty)$, by Lemma 2, we have

$$
\left\|S^{p} T^{q} z-S^{i} T^{j} z\right\| \leqslant\left\|S^{i-p} T^{j-q} z-z\right\| \leqslant d
$$

and hence

$$
\begin{aligned}
f\left(S^{i} T^{j} z-z\right) & =f\left(S^{i} T^{j} z-S^{p} T^{q} z\right)+f\left(S^{p} T^{q} z-z\right) \\
& \geqslant-\|f\| \cdot\left\|S^{i} T^{j} z-S^{p} T^{q} z\right\|+d-\delta \\
& \geqslant-\delta .
\end{aligned}
$$

Putting $A_{n}=A(n, f, \varepsilon) \cap N(p+1, n) \times N(q+1, n)$ for $n \in \mathbb{N}$ with $n>\max \{p, q\}$, since

$$
\begin{aligned}
N(1, n)^{2}=\left(N(p+1, n) \times N(q+1, n) \backslash A_{n}\right) & \sqcup A_{n} \\
& \sqcup\left(N(1, n)^{2} \backslash(N(p+1, n) \times N(q+1, n))\right),
\end{aligned}
$$

we have

$$
\begin{aligned}
f(M(n, z)-z) & =\frac{1}{n^{2}} \sum_{i=1}^{n} \sum_{j=1}^{n} f\left(S^{i} T^{j} z-z\right) \\
& \geqslant \varepsilon \frac{(n-p)(n-q)-\sharp A_{n}}{n^{2}}+(-\delta) \frac{\| A_{n}}{n^{2}}+(-d-\delta) \frac{n^{2}-(n-p)(n-q)}{n^{2}}
\end{aligned}
$$




$$
=\varepsilon \frac{(n-p)(n-q)}{n^{2}}-(\varepsilon+\delta) \frac{\sharp A_{n}}{n^{2}}+(-d-\delta) \frac{n^{2}-(n-p)(n-q)}{n^{2}}
$$

and hence

$$
\liminf _{n \rightarrow \infty} \frac{\sharp A(n, f, \varepsilon)}{n^{2}} \geqslant \liminf _{n \rightarrow \infty} \frac{\sharp A_{n}}{n^{2}} \geqslant \frac{\varepsilon}{\varepsilon+\delta} .
$$

We obtain the desired result.

LEMMA 4.

$$
\lim _{n \rightarrow \infty} \frac{\sharp B(n, \varepsilon)}{n^{2}}=1
$$

hold for all $\varepsilon>0$.

ProOF: We fix $\varepsilon>0$ and $\eta \in \mathbb{R}$ with $1 / 2<\eta<1$ and put

$$
\delta=\frac{\varepsilon(1-\eta)}{2 \eta}
$$

We note that $0<\delta<\varepsilon / 2$. By the definition of $d$, there exists $(p, q) \in \mathbb{N}^{2}$ such that

$$
\left\|S^{p} T^{q} z-z\right\| \geqslant d-\delta
$$

Fix $f \in E^{*}$ with

$$
\|f\|=1 \quad \text { and } \quad f\left(S^{p} T^{q} z-z\right)=\left\|S^{p} T^{q} z-z\right\| .
$$

So, using Lemma 3 , we have

$$
\liminf _{n \rightarrow \infty} \frac{\sharp A(n, f, \varepsilon / 2)}{n^{2}} \geqslant \frac{\varepsilon / 2}{\varepsilon / 2+\delta}=\eta .
$$

Fix $n>\max \{p, q\}$ and $(i, j) \in A(n, f, \varepsilon / 2) \cap(N(p, n) \times N(q, n))$. Then we have

$$
\begin{aligned}
\left\|S^{i-p} T^{j-q} z-z\right\| & \geqslant\left\|S^{i} T^{j} z-S^{p} T^{q} z\right\| \\
& \geqslant f\left(S^{p} T^{q} z-S^{i} T^{j} z\right) \\
& =f\left(S^{p} T^{q} z-z\right)+f\left(z-S^{i} T^{j} z\right) \\
& =\left\|S^{p} T^{q} z-z\right\|+f\left(z-S^{i} T^{j} z\right) \\
& \geqslant d-\delta-\frac{\varepsilon}{2} \\
& \geqslant d-\varepsilon
\end{aligned}
$$

and hence $(i-p, j-q) \in B(n, \varepsilon)$. So, we obtain

$$
\begin{aligned}
\sharp A(n, f, \varepsilon / 2)+(n-p+1)(n-q+1)-n^{2} & \leqslant \sharp(A(n, f, \varepsilon / 2) \cap(N(p, n) \times N(q, n))) \\
& \leqslant \sharp B(n, \varepsilon) .
\end{aligned}
$$

Therefore we have

$$
\liminf _{n \rightarrow \infty} \frac{\sharp B(n, \varepsilon)}{n^{2}} \geqslant \liminf _{n \rightarrow \infty} \frac{\sharp A(n, f, \varepsilon / 2)+(n-p+1)(n-q+1)-n^{2}}{n^{2}}
$$




$$
\begin{aligned}
& =\liminf _{n \rightarrow \infty} \frac{\sharp A(n, f, \varepsilon / 2)}{n^{2}} \\
& \geqslant \eta .
\end{aligned}
$$

Since $\eta$ is arbitrary, we obtain the desired result.

Proof of Theorem 4: By the definition of $d$, there exists $\left(p_{1}, q_{1}\right) \in \mathbb{N}^{2}$ such that

$$
\left\|S^{p_{1}} T^{q_{1}} z-z\right\| \geqslant d-\frac{1}{3^{2}}
$$

Fix $f_{1} \in E^{*}$ with

$$
\left\|f_{1}\right\|=1 \quad \text { and } \quad f_{1}\left(S^{p_{1}} T^{q_{1}} z-z\right)=\left\|S^{p_{1}} T^{q_{1}} z-z\right\| .
$$

By Lemma 3, we have

$$
\liminf _{n \rightarrow \infty} \frac{\sharp A\left(n, f_{1},(2 / 3)^{2}\right)}{n^{2}} \geqslant \frac{2^{2}}{2^{2}+1} .
$$

We now define inductively sequences $\left\{p_{n}\right\}$ and $\left\{q_{n}\right\}$ in $\mathbb{N}$ and $\left\{f_{n}\right\}$ in $E^{*}$ such that

$$
\begin{aligned}
\min \left\{p_{k+1}, q_{k+1}\right\} & >\max \left\{p_{k}, q_{k}\right\}, \\
\left\|S^{p_{k+1}} T^{q_{k+1}} z-z\right\| & \geqslant d-\frac{1}{3^{k+2}}, \\
f_{\ell}\left(S^{p_{k+1}} T^{q_{k+1}} z-z\right) & \leqslant \frac{2^{\ell+1}}{3^{\ell+1}} \text { for } \ell \in N(1, k), \\
\left\|f_{k+1}\right\| & =1 \text { and } f_{k+1}\left(S^{p_{k+1}} T^{q_{k+1}} z-z\right)=\left\|S^{p_{k+1}} T^{q_{k+1}} z-z\right\|
\end{aligned}
$$

for all $k \in \mathbb{N}$. Suppose $p_{k}, q_{k} \in \mathbb{N}$ and $f_{k} \in E^{*}$ are known. Since

$$
\begin{aligned}
\liminf _{n \rightarrow \infty} & \frac{\sharp B\left(n, 1 / 3^{k+2}\right)}{n^{2}}+\sum_{\ell=1}^{k} \liminf _{n \rightarrow \infty} \frac{\sharp A\left(n, f_{\ell},(2 / 3)^{\ell+1}\right)}{n^{2}}-k \\
\geqslant & 1+\sum_{\ell=1}^{k} \frac{2^{\ell+1}}{2^{\ell+1}+1}-k \\
& \geqslant 1+\sum_{\ell=1}^{k} \frac{2^{\ell+1}-1}{2^{\ell+1}}-k=1+\sum_{\ell=1}^{k} \frac{-1}{2^{\ell+1}} \\
& >\frac{1}{2}>0
\end{aligned}
$$

we have

$$
N\left(\max \left\{p_{k}, q_{k}\right\}+1, \infty\right)^{2} \cap B\left(\infty, 1 / 3^{k+2}\right) \cap \bigcap_{\ell=1}^{k} A\left(\infty, f_{\ell},(2 / 3)^{\ell+1}\right) \neq \emptyset
$$

by Lemma 1 , that is, there exists $\left(p_{k+1}, q_{k+1}\right)$ in $\mathbb{N}^{2}$ such that $\min \left\{p_{k+1}, q_{k+1}\right\}$ $>\max \left\{p_{k}, q_{k}\right\}$,

$$
\left\|S^{p_{k+1}} T^{q_{k+1}} z-z\right\| \geqslant d-\frac{1}{3^{k+2}} \quad \text { and } \quad f_{\ell}\left(S^{p_{k+1}} T^{q_{k+1}} z-z\right) \leqslant \frac{2^{\ell+1}}{3^{\ell+1}}
$$


for $\ell \in N(1, k)$. Fix $f_{k+1} \in E^{*}$ with

$$
\left\|f_{k+1}\right\|=1 \quad \text { and } \quad f_{k+1}\left(S^{p_{k+1}} T^{q_{k+1}} z-z\right)=\left\|S^{p_{k+1}} T^{q_{k+1}} z-z\right\| .
$$

Note that

$$
\liminf _{n \rightarrow \infty} \frac{\sharp A\left(n, f_{k+1},(2 / 3)^{k+2}\right)}{n^{2}} \geqslant \frac{2^{k+2}}{2^{k+2}+1}
$$

by Lemma 3 . Hence we have defined $\left\{p_{n}\right\},\left\{q_{n}\right\}$ and $\left\{f_{n}\right\}$. Since $C$ is weakly compact, there exist subsequences $\left\{p_{n_{k}}\right\}$ and $\left\{q_{n_{k}}\right\}$ of $\left\{p_{n}\right\}$ and $\left\{q_{n}\right\}$ respectively such that $\left\{S^{p_{n_{k}}} T^{q_{n_{k}}} z\right\}$ converges weakly to some point $u \in C$. If $n_{k}>\ell$, then

$$
f_{\ell}\left(S^{p_{n_{k}}} T^{q_{n_{k}}} z-z\right) \leqslant \frac{2^{\ell+1}}{3^{\ell+1}} .
$$

So we obtain

$$
f_{\ell}(u-z) \leqslant \frac{2^{\ell+1}}{3^{\ell+1}}
$$

for all $\ell \in \mathbb{N}$. Since

$$
\begin{aligned}
\left\|S^{p_{\ell}} T^{q_{\ell}} z-u\right\| & =\left\|f_{\ell}\right\| \cdot\left\|S^{p_{\ell}} T^{q_{\ell}} z-u\right\| \\
& \geqslant f_{\ell}\left(S^{p_{\ell}} T^{q_{\ell}} z-u\right) \\
& =f_{\ell}\left(S^{p_{\ell}} T^{q_{\ell}} z-z\right)+f_{\ell}(z-u) \\
& =\left\|S^{p_{\ell}} T^{q_{\ell}} z-z\right\|+f_{\ell}(z-u) \\
& \geqslant d-\frac{1}{3^{\ell+1}}-\frac{2^{\ell+1}}{3^{\ell+1}}
\end{aligned}
$$

for $\ell \in \mathbb{N}$, we have

$$
\liminf _{\ell \rightarrow \infty}\left\|S^{p} T^{q \ell} z-u\right\| \geqslant d
$$

and hence

$$
\liminf _{k \rightarrow \infty}\left\|S^{p_{n_{k}}} T^{q_{n_{k}}} z-z\right\| \leqslant d \leqslant \liminf _{\ell \rightarrow \infty}\left\|S^{p_{\ell}} T^{q_{\ell}} z-u\right\| \leqslant \liminf _{k \rightarrow \infty}\left\|S^{p_{n_{k}}} T^{q_{n_{k}}} z-u\right\| .
$$

By the Opial property of $E$, we obtain $z=u$. We also have

$$
\liminf _{k \rightarrow \infty}\left\|S^{p_{n_{k}}} T^{q_{n_{k}}} z-S z\right\| \leqslant \liminf _{k \rightarrow \infty}\left\|S^{p_{n_{k}}-1} T^{q_{n_{k}}} z-z\right\| \leqslant d
$$

and hence $S z=u$. Similarly we can prove $T z=u$. Therefore $z=S z=T z=u$ and we complete the proof.

REMARK . In Theorem 4, we may replace the condition " $E$ has the Opial property" with the following condition: for each weakly convergent sequence $\left\{x_{n}\right\}$ in $C$ with weak limit $z$,

$$
\liminf _{n \rightarrow \infty}\left\|x_{n}-z\right\|<\liminf _{n \rightarrow \infty}\left\|x_{n}-x\right\|
$$


holds for all $x \in C$ with $x \neq z$. If $C$ is compact, then the above condition is satisfied. Indeed, if a sequence $\left\{x_{n}\right\}$ in $C$ converges weakly to $z$, then $\left\{x_{n}\right\}$ converges strongly to $z$. Hence the following holds.

$$
\lim _{n \rightarrow \infty}\left\|x_{n}-z\right\|=0<\|z-x\|=\lim _{n \rightarrow \infty}\left\|x_{n}-x\right\|
$$

for all $x \in E$ with $x \neq z$. So we can prove the following, which is proved in [32].

THEOREM 5. (Suzuki [32].) Let $C$ be a compact convex subset of a Banach space $E$ and let $S$ and $T$ be nonexpansive mappings on $C$ with $S T=T S$. Then for $z \in C$, the following are equivalent:

(i) $z$ is a common fixed point of $S$ and $T$;

(ii)

$$
\liminf _{n \rightarrow \infty}\left\|\frac{1}{n^{2}} \sum_{i=1}^{n} \sum_{j=1}^{n} S^{i} T^{j} z-z\right\|=0
$$

holds.

\section{NONEXPANSIVE RETRACTION}

In this section, we discuss the nonexpansive retraction onto the set of the common fixed points of nonexpansive mappings. Let $S$ and $T$ be nonexpansive mappings on a weakly compact convex subset $C$ of a Banach space $E$. Define $M(\cdot, \cdot)$ by $(1)$. We now define a nonexpansive mapping $U$ on $C$ by using the notion of universal net. A net $\left\{x_{\beta}: \beta \in D\right\}$ in a topological space $X$ is called a universal net [20] if for each subset $A$ of $X$, there exists $\beta_{0} \in D$ such that either $\left\{x_{\beta}: \beta \geqslant \beta_{0}\right\} \subset A$ or $\left\{x_{\beta}: \beta \geqslant \beta_{0}\right\} \subset X \backslash A$ holds. We note that if $X$ is compact, then $\left\{x_{\beta}\right\}$ always converges. From the Axiom of Choice, there exist a directed set $(D, \leqslant)$ and a universal subnet $\left\{\nu_{\beta}: \beta \in D\right\}$ in $\mathbb{N}$ of a sequence $\{n: n \in \mathbb{N}\}$ in $\mathbb{N}$, that is,

(i) for each $n \in \mathbb{N}$ there exists $\beta_{0} \in D$ such that $\beta \geqslant \beta_{0}$ implies $\nu_{\beta} \geqslant n$; and

(ii) for each subset $A$ of $\mathbb{N}$, there exists $\beta_{0} \in D$ such that either $\left\{\nu_{\beta}: \beta\right.$ $\left.\geqslant \beta_{0}\right\} \subset A$ or $\left\{\nu_{\beta}: \beta \geqslant \beta_{0}\right\} \subset \mathbb{N} \backslash A$ holds.

For $x \in C$, since $\left\{\nu_{\beta}: \beta \in D\right\}$ is a universal net, $\left\{M\left(\nu_{\beta}, x\right): \beta \in D\right\}$ is also a universal net in $C$. Hence, by the weak compactness of $C,\left\{M\left(\nu_{\beta}, x\right)\right\}$ converges weakly. Now, we define $U x$ as

$$
U x=\underset{\beta \in D}{\operatorname{weak}} \lim M\left(\nu_{\beta}, x\right)
$$

for all $x \in C$. Such $U$ is a nonexpansive mapping on $C$ because

$$
\|U x-U y\| \leqslant \liminf _{\beta \in D}\left\|M\left(\nu_{\beta}, x\right)-M\left(\nu_{\beta}, y\right)\right\| \leqslant\|x-y\|
$$

for $x, y \in C$. 
THEOREM 6. Assume that either of the following holds:

(i) $E$ is a Banach space with the Opial property and $C$ is a weakly compact convex subset of $E$; or

(ii) $E$ is a Banach space and $C$ is a compact convex subset of $E$.

Let $S$ and $T$ be nonexpansive mappings on $C$ with $S T=T S$. Define a nonexpansive mapping $U$ on $C$ by (2). Then $F(S) \cap F(T)=F(U)$ holds.

Proof: We first assume $S z=T z=z$. Then $M(n, z)=z$ for all $n \in \mathbb{N}$ and hence $U z=z$. Conversely, we next assume $U z=z$. Then in the case of (i), since

$$
z=U z=\underset{\beta \in D}{\operatorname{weak}-\lim } M\left(\nu_{\beta}, z\right)
$$

we obtain $S z=T z=z$ by Theorem 4 . In the case of (ii), we also have

$$
\liminf _{n \rightarrow \infty}\|M(n, z)-z\| \leqslant \lim _{\beta \in D}\left\|M\left(\nu_{\beta}, z\right)-z\right\|=0 .
$$

So, by Theorem 5 , we obtain $S z=T z=z$.

Using Theorem 6 , we obtain the following convergence theorems to a common fixed point of nonexpansive mappings.

TheOREM 7. Let $E$ be a Banach space with the Opial property and let $C$ be a weakly compact convex subset of $E$. Let $S$ and $T$ be nonexpansive mappings on $C$ with $S T=T S$. Define a nonexpansive mapping $U$ on $C$ by (2). Let $x_{1} \in C$ and define a sequence $\left\{x_{n}\right\}$ in $C$ by

$$
x_{n+1}=\alpha U x_{n}+(1-\alpha) x_{n}
$$

for $n \in \mathbb{N}$, where $\alpha$ is a constant number in $(0,1)$. Then $\left\{x_{n}\right\}$ converges weakly to a common fixed point $z_{0}$ of $S$ and $T$.

Proof: By Theorem 2, $\left\{x_{n}\right\}$ converges weakly to a fixed point $z_{0}$ of $U$, which is a common fixed point of $S$ and $T$, by Theorem 6 . This completes the proof.

THEOREM 8. Let $C$ be a compact convex subset of a Banach space $E$ and let $S$ and $T$ be nonexpansive mappings on $C$ with $S T=T S$. Define a nonexpansive mapping $U$ on $C$ by (2). Let $x_{1} \in C$ and define a sequence $\left\{x_{n}\right\}$ in $C$ by

$$
x_{n+1}=\alpha U x_{n}+(1-\alpha) x_{n}
$$

for $n \in \mathbb{N}$, where $\alpha$ is a constant number in $(0,1)$. Then $\left\{x_{n}\right\}$ converges strongly to a common fixed point $z_{0}$ of $S$ and $T$.

Proof: By Theorem 3, $\left\{x_{n}\right\}$ converges strongly to a fixed point $z_{0}$ of $U$, which is a common fixed point of $S$ and $T$, by Theorem 6 . This completes the proof.

Using Theorems 7 and 8 , we prove the existence theorem. 
THEOREM 9. Assume that either of the following holds:

(i) $E$ is a Banach space with the Opial property and $C$ is a weakly compact convex subset of $E$; or

(ii) $E$ is a Banach space and $C$ is a compact convex subset of $E$.

Let $S$ and $T$ be nonexpansive mappings on $C$ with $S T=T S$. Then there exists a nonexpansive retraction $Q$ from $C$ onto $F(S) \cap F(T)$ such that $Q=Q^{2}=Q S=S Q$ $=Q T=T Q$.

Proof: We first define a mapping $Q$ on $C$. Fix $x \in C$. Define a sequence $\left\{x_{n}\right\}$ by $x_{1}=U x$ and

$$
x_{n+1}=\frac{1}{2} U x_{n}+\frac{1}{2} x_{n}
$$

for $n \in \mathbb{N}$. where $U$ is a nonexpansive mapping on $C$ defined by (2). Then as the proofs of Theorems 7 and $8,\left\{x_{n}\right\}$ converges weakly to a common fixed point $z_{0}$ of $S$ and $T$. We put $Q x=z_{0}$, that is,

$$
Q x=\underset{n \rightarrow \infty}{\text { weak- } \lim }\left(\frac{1}{2} U+\frac{1}{2} I\right)^{n} U x,
$$

where $I$ is the identity mapping on $C$. Since $x \in C$ is arbitrary, we have defined the mapping $Q$ on $C$. For $x, y \in C$, we have

$$
\|Q x-Q y\| \leqslant \liminf _{n \rightarrow \infty}\left\|\left(\frac{1}{2} U+\frac{1}{2} I\right)^{n} U x-\left(\frac{1}{2} U+\frac{1}{2} I\right)^{n} U y\right\| \leqslant\|U x-U y\| \leqslant\|x-y\|
$$

and hence $Q$ is nonexpansive. For $x \in C$, we also have

$$
\begin{aligned}
\|U S x-U x\| & \leqslant \liminf _{\beta}\left\|M\left(\nu_{\beta}, S x\right)-M\left(\nu_{\beta}, x\right)\right\| \\
& =\liminf _{\beta}\left\|\frac{1}{\nu_{\beta}^{2}} \sum_{j=1}^{\nu_{\beta}}\left(S^{\nu_{\beta}+1} T^{j} x-S T^{j} x\right)\right\| \\
& \leqslant \liminf _{\beta} \frac{1}{\nu_{\beta}^{2}} \sum_{j=1}^{\nu_{\beta}}\left\|S^{\nu_{\beta}+1} T^{j} x-S T^{j} x\right\| \\
& \leqslant \liminf _{\beta} \frac{1}{\nu_{\beta}}\left\|S^{\nu_{\beta}} x-x\right\| \\
& =0
\end{aligned}
$$

and hence $U S x=U x$. Therefore $Q S x=Q x$ for all $x \in C$. Similarly we can prove $Q T x=Q x$. By the definition of $Q, Q x \in F(S) \cap F(T)$ for all $x \in C$. From Theorem 6, it is clear that $Q z=z$ for all $z \in F(S) \cap F(T)$. Hence we also have $Q^{2} x=Q x=S Q x=T Q x$ for all $x \in C$. This completes the proof.

REMARK. (i) In the case that $E$ is a Hilbert space, $\{M(n, x)\}$ converges weakly to a common fixed point of $S$ and $T$ for all $x \in C$; see $[34$, p. 83]. Therefore a nonexpansive 
mapping $U$ itself is a nonexpansive retraction from $C$ onto $F(S) \cap F(T) . U$ also satisfies $U S=U T=U$. Therefore the following question arise: In our setting, is $U$ always a nonexpansive retraction onto $F(S) \cap F(T)$ ? The answer is negative; see Example 2 in Section 5 .

(ii) Gossez and Lami Dazo [16] prove that every weakly compact convex subset of a Banach space with the Opial property has normal structure. So, Bruck's result (Theorem 1) shows that there exists a nonexpansive retraction $Q$ from $C$ onto $F(S) \cap$ $F(T)$. However, Theorem 1 does not assure $Q S=Q T=Q$.

As a direct consequence of Theorem 9, we obtain the following.

Corollary 1. Assume that either of the following holds:

(i) $E$ is a Banach space with the Opial property and $C$ is a weakly compact convex subset of $E$; or

(ii) $E$ is a Banach space and $C$ is a compact convex subset of $E$.

Let $T$ be nonexpansive mappings on $C$. Then there exists a nonexpansive retraction $Q$ from $C$ onto $F(T)$ such that $Q=Q^{2}=Q T=T Q$.

\section{ApPEndix}

In this section we give two examples. We first state an example concerning Theorem 4.

EXAMPLE 1. ([14, Example 3.7].) Let $c_{0}$ be the Banach space consisting of all sequences in $\mathbb{R}$ converging to 0 with supremum norm. Define nonexpansive mappings $S$ and $T$ on $c_{0}$ by

$$
S x=x \quad \text { and } T\left(x_{1}, x_{2}, x_{3}, \ldots\right)=\left(x_{1}, 1-\|x\|, x_{2}, x_{3}, x_{4}, \ldots\right)
$$

for all $x=\left(x_{1}, x_{2}, x_{3}, \ldots\right) \in c_{0}$. Then $0 \in c_{0}$ is not a fixed point of $T$ and

$$
\lim _{n \rightarrow \infty}\left\|\frac{1}{n^{2}} \sum_{i=1}^{n} \sum_{j=1}^{n} S^{i} T^{j} 0-0\right\|=0
$$

holds.

Proof: It is clear that

$$
\|S x-S y\|=\|T x-T y\|=\|x-y\|
$$

for all $x, y \in c_{0}$ and hence $S$ and $T$ are nonexpansive. We put

$$
e_{k}=(0,0,0, \ldots, 0, \stackrel{(k)}{1}, 0,0,0, \ldots) \in c_{0}
$$

for $k \in \mathbb{N}$. Then the following hold:

$$
T 0=e_{2},
$$




$$
\begin{aligned}
T e_{1} & =e_{1}, \\
T e_{2} & =e_{3}, \\
& \vdots \\
T e_{k} & =e_{k+1} .
\end{aligned}
$$

We suppose that $x=\left(x_{1}, x_{2}, x_{3}, \ldots\right) \in c_{0}$ satisfies $T x=x$. Then we have $x_{2}=x_{3}=x_{4}$ $=\cdots$ and hence

$$
x_{2}=x_{3}=x_{4}=\ldots=0
$$

because $\left\{x_{n}\right\}$ converges to 0 . Therefore $\|x\|=\left|x_{1}\right|$. So, we have

$$
0=x_{2}=1-\|x\|=1-\left|x_{1}\right|
$$

and hence $x_{1}= \pm 1$, that is, $x=e_{1}$ or $x=-e_{1}$. Conversely, we also have $T e_{1}=e_{1}$ and $T\left(-e_{1}\right)=-e_{1}$. Therefore

$$
F(S) \cap F(T)=F(T)=\left\{e_{1},-e_{1}\right\} .
$$

This shows that 0 is not a common fixed point of $S$ and $T$. Since $S^{i} T^{j} 0=e_{j+1}$ for $i, j \in \mathbb{N}$, we have

$$
\lim _{n \rightarrow \infty}\left\|\frac{1}{n^{2}} \sum_{i=1}^{n} \sum_{j=1}^{n} S^{i} T^{j} 0-0\right\|=\lim _{n \rightarrow \infty}\left\|\frac{1}{n} \sum_{j=1}^{n} e_{j+1}\right\|=\lim _{n \rightarrow \infty} \frac{1}{n}=0 .
$$

This completes the proof.

We finally state a counterexample concerning the remark of Theorem 9 .

Example 2. ([33, Theorem 15].) Define a compact convex subset $C$ of $\left(\mathbb{R}^{2},\|\cdot\|_{\infty}\right)$ by

$$
C=\left\{\left(x_{1}, x_{2}\right): 0 \leqslant x_{2} \leqslant 1,-x_{2} \leqslant x_{1} \leqslant x_{2}\right\} .
$$

Define nonexpansive mappings $S$ and $T$ on $C$ by

$$
S x=x \quad \text { and } \quad T\left(x_{1}, x_{2}\right)=\left(-x_{1},\left|x_{1}\right|\right)
$$

for all $x=\left(x_{1}, x_{2}\right) \in C$. Then the nonexpansive mapping $U$ defined by (2) is not a nonexpansive retraction from $C$ onto $F(S) \cap F(T)$, and

$$
Q(1,1) \notin \operatorname{clco}\left\{S^{i} T^{j}(1,1): i, j \in \mathbb{N} \cup\{0\}\right\},
$$

where $Q$ is the nonexpansive retraction from $C$ onto $F(S) \cap F(T)$ defined by (3), and 'clco' means the closed convex hull. 
Proof: Since

$$
\begin{aligned}
\left\|M(n, x)-\left(0,\left|x_{1}\right|\right)\right\| & =\left\|\frac{1}{n^{2}} \sum_{i=1}^{n} \sum_{j=1}^{n} S^{i} T^{j} x-\left(0,\left|x_{1}\right|\right)\right\| \\
& =\left\|\frac{1}{n} \sum_{j=1}^{n} T^{j} x-\left(0,\left|x_{1}\right|\right)\right\| \\
& =\left\|\left(\frac{1}{n} \sum_{j=1}^{n}(-1)^{j} x_{1},\left|x_{1}\right|\right)-\left(0,\left|x_{1}\right|\right)\right\| \\
& \leqslant \frac{1}{n}\left|x_{1}\right|,
\end{aligned}
$$

we have $\lim _{n} M(n, x)=\left(0,\left|x_{1}\right|\right)$ for all $x=\left(x_{1}, x_{2}\right) \in C$. Therefore

$$
U\left(x_{1}, x_{2}\right)=\left(0,\left|x_{1}\right|\right)
$$

for all $x=\left(x_{1}, x_{2}\right) \in C$. From $U(1,1)=(0,1)$ and $T(0,1)=(0,0), U$ is not a nonexpansive retraction onto the set of common fixed points of $S$ and $T$. Since $F(S) \cap F(T)=\{(0,0)\}, Q x=(0,0)$ for all $x \in C$, and hence $Q(1,1)=(0,0)$. On the other hand,

$$
\operatorname{clco}\left\{S^{i} T^{j}(1,1): i, j \in \mathbb{N} \cup\{0\}\right\}=\left\{\left(x_{1}, 1\right):-1 \leqslant x_{1} \leqslant 1\right\} .
$$

This completes the proof.

\section{REFERENCES}

[1] S. Atsushiba and W. Takahashi, 'Approximating common fixed points of two nonexpansive mappings in Banach spaces', Bull. Austral. Math. Soc. 57 (1998), 117-127.

[2] L.P. Belluce and W.A. Kirk, 'Fixed-point theorems for families of contraction mappings', Pacific J. Math. 18 (1966), 213-217.

[3] L.P. Belluce and W.A. Kirk, 'Nonexpansive mappings and fixed-points in Banach spaces', Ilinois J. Math 11 (1967), 474-479.

[4] F.E. Browder, 'Nonexpansive nonlinear operators in a Banach space', Proc. Nat. Acad. Sci. U.S.A. 54 (1965), 1041-1044.

[5] R.E. Bruck, 'Nonexpansive retracts of Banach spaces', Bull. Amer. Math. Soc. 76 (1970), 384-386.

[6] R.E. Bruck, 'Properties of fixed-point sets of nonexpansive mappings in Banach spaces', Trans. Amer. Math. Soc. 179 (1973), 251-262.

[7] R.E. Bruck, 'A common fixed point theorem for a commuting family of nonexpansive mappings', Pacific J. Math. 53 (1974), 59-71.

[8] G. Crombez, 'Image recovery by convex combinations of projections', J. Math. Anal. Appl. 155 (1991), 413-419.

[9] R. DeMarr, 'Common fixed points for commuting contraction mappings', Pacific J. Math. 13 (1963), 1139-1141. 
[10] D. van Dulst, 'Equivalent norms and the fixed point property for nonexpansive mappings', J. London Math. Soc. (2) 25 (1982), 139-144.

[11] M. Edelstein, 'A remark on a theorem of M.A. Krasnoselski', Amer. Math. Monthly 73 (1966), 509-510.

[12] M. Edelstein and R.C. O'Brien, 'Nonexpansive mappings, asymptotic regularity and successive approximations', J. London Math. Soc. (2) 17 (1978), 547-554.

[13] D.G. de Figueiredo and L.A. Karlovitz, 'On the extension of contractions on normed spaces', in 1970 Nonlinear Functional Analysis, Proc. Sympos. Pure Math., Vol. XVIII, Part 1, Chicago, Ill., 1968 (American Mathematical Society, Providence, R.I), pp. 95-104.

[14] K. Goebel and W.A. Kirk, Topics in metric fixed point theory, Cambridge Studies in Advanced Mathematics 28 (Cambridge University Press, Cambridge, 1990).

[15] D. Göhde, 'Zum Prinzip def kontraktiven Abbildung', Math. Nachr. 30 (1965), 251-258.

[16] J.-P. Gossez and E. Lami Dazo, 'Some geometric properties related to the fixed point theory for nonexpansive mappings', Pacific J. Math. 40 (1972), 565-573.

[17] C.W. Groetsch, 'A note on segmenting Mann iterates', J. Math. Anal. Appl. 40 (1972), 369-372.

[18] S. Ishikawa, 'Fixed points and iteration of a nonexpansive mapping in a Banach space', Proc. Amer. Math. Soc. 59 (1976), 65-71.

[19] S. Ishikawa, 'Common fixed points and iteration of commuting nonexpansive mappings', . Pacific J. Math. 80 (1979), 493-501.

[20] J.L. Kelley, General topology (Van Nostrand Reinhold Company, Toronto, New York, London, 1955).

[21] W.A. Kirk, 'A fixed point theorem for mappings which do not increase distances', Amer. Math. Monthly 72 (1965), 1004-1006.

[22] M.A. Krasnoselskii, 'Two remarks on the method of successive approximations', (in Russian), Uspehi Mat. Nauk 10 (1955), 123-127.

[23] E. Lami Dazo, 'Multivalued nonexpansive mappings and Opial's condition', Proc. Amer. Math. Soc. 38 (1973), 286-292.

[24] T.C. Lim, 'A fixed point theorem for families of nonexpansive mappings', Pacific J. Math 53 (1974), 487-493.

[25] P.-K. Lin, K.K. Tan and H.K. Xu, 'Demiclosedness principle and asymptotic behavior for asymptotically nonexpansive mappings', Nonlinear Anal. 24 (1995), 929-946.

[26] J. Linhart, 'Beiträge zur Fixpunkttheorie nichtexpandierender Operatoren', Monatsh. Math. 76 (1972), 239-249.

[27] W.R. Mann, 'Mean value methods in iteration', Proc. Amer. Math. Soc. 4 (1953), 506-510.

[28] Z. Opial, 'Weak convergence of the sequence of successive approximations for nonexpansive mappings', Bull. Amer. Math. Soc. 73 (1967), 591-597.

[29] S. Prus, 'Banach spaces with the uniform Opial property', Nonlinear Anal. 18 (1992), 697-704.

[30] S. Reich, 'Weak convergence theorems for nonexpansive mappings', J. Math. Anal. Appl. 67 (1979), 274-276.

[31] B. Sims, 'A support map characterization of the Opial conditions', Proc. Centre Math. Anal. Austral. Nat. Univ. 9, 259-264. 
[32] T. Suzuki, 'Strong convergence theorem to common fixed points of two nonexpansive mappings in general Banach spaces', J. Nonlinear Convex Anal. 3 (2002), 381-391.

[33] T. Suzuki and W. Takahashi, 'Weak and strong convergence theorems for nonexpansive mappings in Banach spaces', Nonlinear Anal. 47 (2001), 2805-2815.

[34] W. Takahashi, Nonlinear functional analysis (Yokohama Publishers, Yokohama, 2000).

Department of Mathematics and Information Science

Graduate School of Science and Technology

Niigata University

Niigata 950-2181

Japan

e-mail: tomonari@math.sc.niigata-u.ac.jp

Present address:

Department of Mathematics

Kyushu Institute of Technology

1-1 Sensuicho Tobata-ku

Kitakyushu 804-8550

Japan

e-mail: suzuki-t@mns.kyutech.ac.jp 